\title{
Position Tracking for Outdoor Sport Events with GNSS and LoRa
}

\author{
Juan-Mario Gruber ${ }^{1}$, Benjamin Brossi ${ }^{1}$ \\ ${ }^{1}$ Institute of Embedded Systems (InES) \\ Zurich University of Applied Sciences (ZHAW) \\ 8401Winterthur, Switzerland \\ gruj@zhaw.ch, broi@zhaw.ch
}

\begin{abstract}
Outdoor sport events often require a real time tracking of the position of participants or equipment within a defined area in real time. Global navigation satellite systems (GNSS) determine the position with great accuracy. In addition, using the LoRa radio technology, data is transmitted under optimal conditions over a distance up to $4 \mathrm{~km}$.
\end{abstract}

Key words: LoRa; GNSS; Real-Time Position Tracking; Low Power; Energy Harvesting

\section{Motivation}

Outdoor sport events often need position tracking of participants.. Since the participants are often spread over a wide area, the data must be able to be transmitted over a large distance.

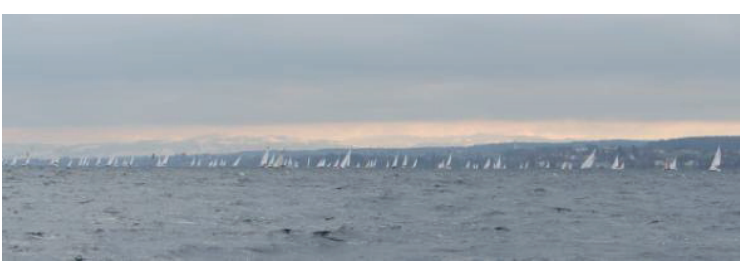

Fig. 1. Position tracking at a sailing regatta

A new system has been developed to transmit real-time position data from a global navigation satellite system (GNSS) live over a long distance to a base station. The base station visualizes the data on a map. By defining a finish line, the crossing of the finish line can be detected. For data transmission the LoRa wireless standard is used. The whole system is to be optimized for low energy consumption, so that it can be operated for several hours by rechargeable batteries or energy harvesting. In summary, the following objectives can be defined:

The system is optimized for low energy consumption, live data from a GNSS is used, the data is transmitted via LoRa, the trackers are inexpensive to produce and the position data is displayed in real time.

\section{Concept}

The system tracks the position of up to 255 independent objects in a large-scale area. It consists of multiple position trackers and a central base station (Position Tracker Manager).

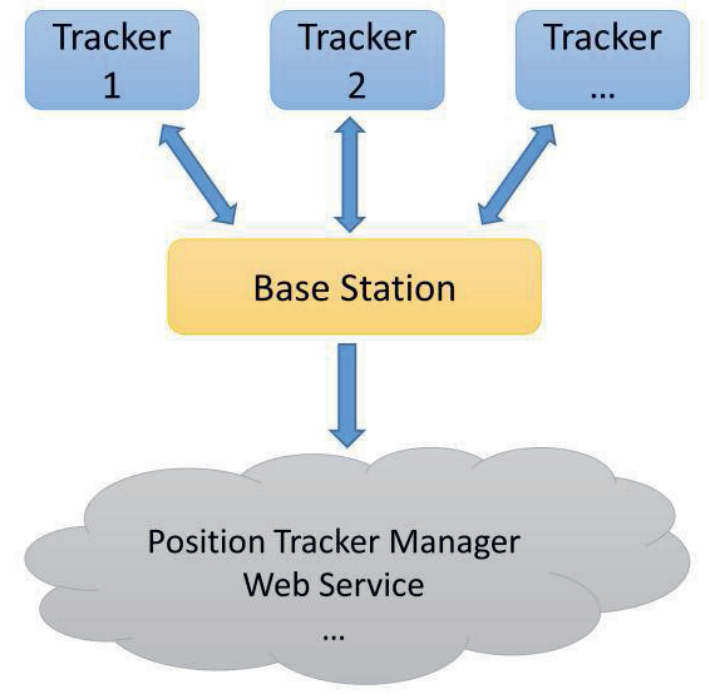

Fig. 2. System block diagram [1]

The system implements a standard LoRa functionality and uses the $868 \mathrm{MHz}$ band. The position trackers are built with latest very low power components and are optimized for low power consumption. This means that they are ready to be powered by energy harvesting.

The system communicates bidirectionally. Thus the base station can configure and control the 
trackers at every time. The data package consists of object number, position data and time stamps. This has to be done because only a certain amount of data per hour can be sent through the LoRa band. The system can automatically perform position-based evaluations such as crossing the finish line.

\section{Tracker device}

The tracker devices (Fig. 3) use GNSS to determine time and position data send them via LoRa.

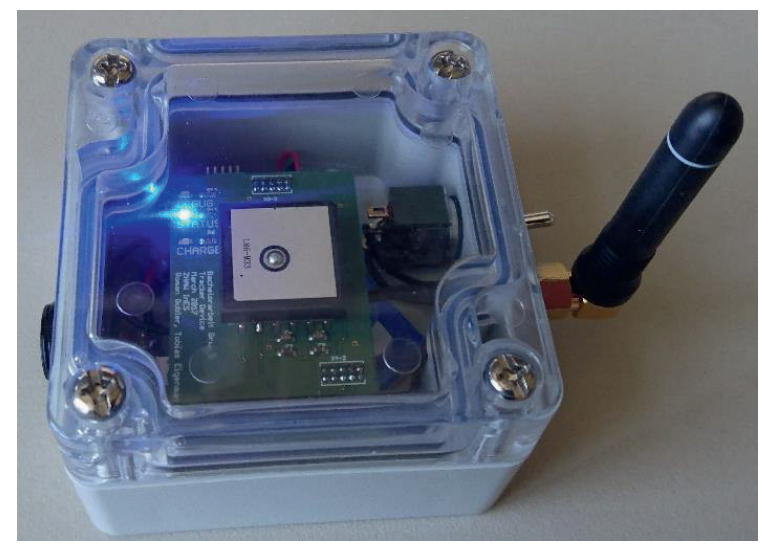

Fig. 3. Tracker device prototype

The GNSS module L86-M33 from Quectel is used to determine the time and position. GPS, GLONASS and QZSS can be used with this module. The LoRa module iM880B-L from IMST is used for the data transfer. This is a certified module for wireless communication via the LoRa radio standard. The module features a STM32L151 microcontroller with an ARM Cortex M3 core and a SX1272 LoRa chip from Semtech.

Fig. 4 shows the software state diagram for the tracking device. When switching on the track device, the required peripheral modules of the microcontroller, the LoRa radio and the GNSS module are initialized first. After the initialization, a valid time of the GNSS module is waited for and then the RTC is synchronized to this time. This may take a few minutes, depending on the signal quality of the satellite signals. When the RTC is successfully synchronized, the log and TDMA counters are started. The software changes to idle state.

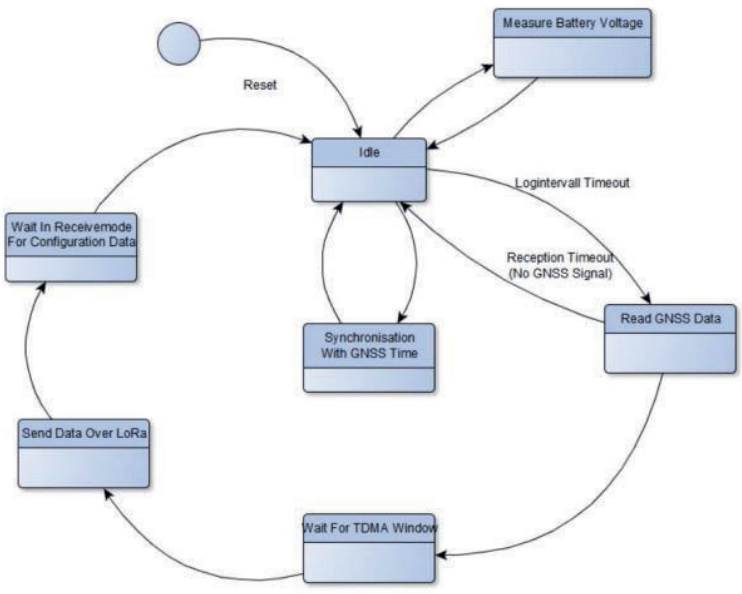

Fig. 4. State diagram tracker device

The software changes to the log state by an event, in which the UART is set to receive the data of the GNSS module. If the data has been successfully received, the data is buffered. The data in the buffer is sent to the base station via LoRa. When transmission is complete, the software switches to receive mode in which it is possible to receive configuration data from the Base Station. The reception time window is 30 milliseconds according to the TDMA protocol. If configuration data is received, the new log interval is set and the log and TDMA counters are restarted.

If a low battery event occurs, the log and TDMA counters are deactivated. An attempt is made to determine a last position within 30 seconds. If successful, this position is transmitted in Low Power Mode. All components are then switched to the low power mode in order to consume as little power as possible. The Tracker Device must then be recharged and restarted with the switch.

For the transmission the smallest possible log interval has to be determined. Also the sending interval for the data packets is to be defined. In order to increase the reliability of data transmission, the current and previous position data are transmitted during each transmission. It turned out that the best results are achieved with 3 seconds log interval and 9 seconds transmission interval.

\section{Base station}

The base station uses a Raspberry $\mathrm{Pi}$ with a LoRa hat. The Raspberry is running a data logger and provides data for the web interface and for the Position Tracker Manager.

When the base station is turned on, it is in the idle state. Receiving data triggers an interrupt. The system checks whether the data is valid. If this is the case, the new log interval is saved so that it can be configured via LoRa when 
receiving data from the corresponding tracker device.

The system communicates bidirectionally, so if data has been received, the software switches to transmit mode in which the new log interval is sent to the tracker device, if a new log interval has to be configured. If there is no new log interval, the software switches to the data processing mode. The data received via LoRa are stored in this module. In this state the times of the positions are reconstructed, assigned to the corresponding tracker device ID and stored. The system then uses the time to check whether the position has already been received. If this is not the case, the position is sent via the UART interface. The software then changes back to the idle state.

\section{PC software (Position Tracker Manager)}

The base station is connected to the Position Tracker Manager via a virtual COM interface via USB. The data is transferred to the computer via UART. The Position Tracker Manager evaluates the data and displays the device trackers on an embedded map. In the software, two trackers can be defined as start and end points of the finish line. The software automatically calculates the distance to the finish line and detects when it is crossed. The transferred raw data and the ranking list can be exported from the software into a CSV file for further use.

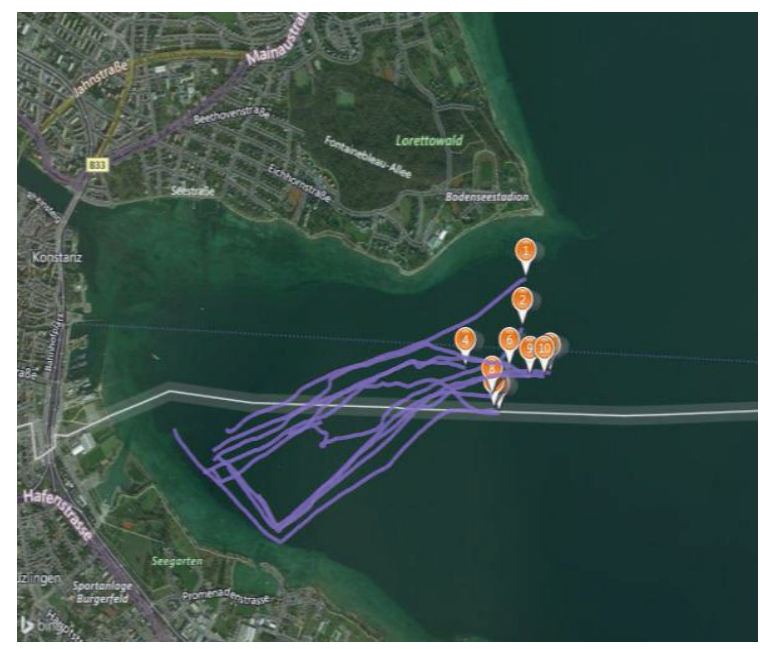

Fig. 5. Life visualization

\section{Energy Consumption}

A Li-lon battery powers the tracker device. Additionally, a charging circuit with integrated buck converter and 3.3 VDC output is used to charge this battery. The charging circuit is supplied by a 5 VDC plug from a power supply unit.

To determine the current consumption of the LoRa module iM880B-L, the following values of the current consumption are taken from the data sheet [2]:

Tab. 1: Current consumption iM880B-L

\begin{tabular}{|c|c|}
\hline State & Current \\
\hline Idle & $5 \mathrm{~mA}$ \\
\hline Transmit & $90 \mathrm{~mA}$ \\
\hline Receive & $11.22 \mathrm{uA}$ \\
\hline $\begin{array}{c}\text { Low Power Mode } \\
\text { RTC ON }\end{array}$ & $1.85 \mathrm{uA}$ \\
\hline $\begin{array}{c}\text { Low Power Mode } \\
\text { RTC OFF }\end{array}$ & $0.8 \mathrm{uA}$ \\
\hline
\end{tabular}

The module can transmit a maximum of 36 seconds per hour at $14 \mathrm{dBm}$ in worst case scenario. This corresponds to the maximum permitted values issued by the Federal Office of Communications (OFCOM). The time for the receiving state is shorter than that of the sending process and in this case is specified at 10 seconds per hour. The remaining time the module is in Low Power Mode RTC ON. These values result in an average current of 0.933 $\mathrm{mA}$.

To calculate the total power consumption of the tracker device, the current consumption of the individual components is added together. Based on the capacity of the battery used, a battery life of $16.6 \mathrm{~h}$ can be estimated [1].

Tab. 2: Current consumption tracker device

\begin{tabular}{|c|c|}
\hline Item & Current \\
\hline Quectel L86-M33 & $26 \mathrm{~mA}$ [2] \\
\hline iM880B-L & $0.933 \mathrm{~mA}$ \\
\hline LEDs & $2 \mathrm{~mA}$ \\
\hline LTC4080 & $1.9 \mathrm{~mA}[3]$ \\
\hline Total & $30.833 \mathrm{~mA}$ \\
\hline Estimated battery life & $\begin{array}{c}500 \mathrm{mAh} / 30.1= \\
16.6 \mathrm{~h}\end{array}$ \\
\hline
\end{tabular}

\section{Test Results}

Field tests at sailing regattas have shown that the device trackers work reliably. The positions are resolved with an accuracy of a few meters or less and the data transfer works in good conditions up to $3 \mathrm{~km}$. The battery life of over 16 hours is sufficient for most sports and competitions. It should also be possible to operate the device trackers with an outdoor solar panel. The device trackers are ideal for use in open terrain. In built-up areas, both the 
accuracy of the position and the maximum transmission distance are reduced.

\section{Conclusion and outlook}

It has been proven that it is possible to operate the device trackers with a very small energy budget. The accuracy of position and transmission distance is sufficient for most applications.

\section{References}

[1] T. Eigenmann, R. Gubler, EchtzeitPositionstracker mit LoRa (Bachelor Thesis, advisor J. Gruber), Zurich University of Applied Science, 2017

[2] iM880B Datasheet, v1.3, IMST GmbH Wireless Solutions, 2016

[3] L86 Hardware Design, Rev. V1.0, Quectel Wireless Solutions, 2014

[4] LTC4080 Datasheet, Rev C, Linear Technology, 2015
In future, the base station will be replaced by a Raspberry $\mathrm{Pi}$ with a LoRa hat. The Raspberry $\mathrm{Pi}$ should be able to perform the data evaluation without the need of a PC. In addition, a web server and a JavaScript application should replace the position Tracker Manager. 